\title{
ОПРЕДЕЛЕНИЕ СВОЙСТВ ОПАСНЫХ СИГНАЛОВ
}

В данной статье приведены результаты исследования подкласса опасных сигналов, допускающих восстановление по заданному модулю спектра с помощью линейных интегральных преобразований и методов перевода сигналов в этот подкласс. Рассмотрены финитные функции, для которых существует неитерационный путь восстановления по модулю преобразования Фурье и комплексном экспоненциальном базисе.

Ключевые слова: опасные сигналы, модификация сигналов.

Введение. В последнее время исследователей интересуют задачи, связанные с нахождением сигналов по известному модулю или квадрату модуля преобразования Фурье этого сигнала. По характеру задача, возникающая при классификации опасных сигналов с охраняемого объекта по спектральным признакам, когда в качестве первичных данных об исследуемых сигналах используются их амплитудные аппаратурные спектры. Частичные решения подобных задач известны и восстановление комплексных сигналов по их Фурье преобразованиям в разных областях техники широко известны. [1] Однако не найдены общие условия, при которых возможно точное решение задачи синтеза сигнала по модулю или аргументу комплексного спектра на основе интегральных преобразований. Анализ свойств преобразования Фурье позволяет сформулировать условия, когда эта задача решается с достаточной для прикладной задачи точностью.

Основная часть. Существует важная для многих практических приложений связь между функциями, равными нулю по полуоси $(-\infty, 0)$ и асимптотическими функциями, интегрируемыми в полуплоскости. В [1] доказана следующая теорема:

Теорема 1. Для того, чтобы комплексная функция $f(\omega) \in L_{2}(-\infty, \infty)$ была пределом при $z \rightarrow \infty$ аналитической в верхней полуплоскости функции $f(z)$, такой, что

$$
\int_{-\infty}^{\infty 2} \mid f\left(\omega+\left.j \eta\right|^{2} d \omega<K\right.
$$

необходимо и достаточно выполнения любого из двух условий:

$$
f(\omega)=g_{1}(\omega)+j g_{2}(\omega)
$$

где $g_{1}$ и $g_{2}-$ сопряжения по Гильберту функции класса $L_{2}$; преобразование Фурье

$f(x)=\frac{1}{2 \pi} \int_{-\infty}^{\infty} f(\omega) e^{-j \omega x} d \omega=0$, при $\mathrm{x}<0$.

Условие (2) позволяет для некоторого класса аналитических функций связать действительную и мнимую части $f(\omega)$. В дальнейшем потребуются теоремы Винера - Пэли, которые в работе [2] обобщаются для функций класса $L_{p}, 1<p<\infty$.

Теорема 2. Преобразование Фурье от ограниченной функции обращается в нуль на интервале $(-\infty, \infty)$ тогда и только тогда, когда $f(\omega)$ есть предел на действительной оси функции $f(z), z=\omega+j \eta$, аналитической в верхней полуплоскости и удовлетворяющей неравенству $\left.\sup |f(\omega+j \eta)| \leq e^{-a \eta} \sup \mid f(\omega)\right], \eta \geq 0$.

Если преобразование Фурье от ограниченной функции $f$ обращается в нуль на интервале $(\beta, \infty)$, то аналогичная теорема утверждает, что $f(\omega)$ является пределом на действительной оси функции $f(z), z=\omega+j \eta$, аналитической в нижней полуплоскости, $\eta \leq 0$.

Теорема 3. Если $f(z)$ - функция, аналитическая в верхней полуплоскости, так, что

$$
\sup |f(\omega+j \eta)|<\infty \text { для } \eta \geq 0,
$$


и имеет место асимптотическая оценка

$$
\sup |f(\omega+j \eta)|=0\left\{e^{-\alpha \eta}\right\}, \eta \rightarrow \infty,
$$

то справедливо неравенство

$$
\sup |f(\omega+j \eta)|<e^{-\alpha \eta} \sup |f(\omega)|, \eta \geq 0
$$

Доказательство теорем 2 и 3 приводится в работе [2].

В многих приложениях, а особенно в радиомониторинге опасных сигналов с защищенных объектов, нужно связать модуль и аргумент комплексной функции $f(\omega)$, которая является преобразованием Фурье некоторой действительной функции $\tilde{f}(x)$. Рассмотрим функцию

$$
F(\omega)=\operatorname{Ln} \tilde{f}(\omega)=\ln |\tilde{f}(\omega)|+j \arg \tilde{f}(\omega)
$$

Если $F(\omega)$ удовлетворяет условиям теоремы 2, то логарифм модуля и аргумент $f(\omega)$ оказывается связанным преобразования Гильберта

$$
\begin{aligned}
& \arg f(\omega)=-\frac{1}{\pi} P \int_{-\infty}^{\infty} \frac{\ln |f(\xi)|}{\xi-\omega} d \xi ; \\
& \ln |f(\omega)|=-\frac{1}{\pi} P \int_{-\infty}^{\infty} \frac{\arg |f(\xi)|}{\xi-\omega} d \xi,
\end{aligned}
$$

где $\boldsymbol{P}$ обозначает главное значение интеграла при $\xi=\omega$.

Определяем условия, которым должны удовлетворять функция $f(x)$ и ее преобразование Фурье $f(\omega)$, чтобы выражения (7) и (8) можно было использовать для определения $f(\omega)$ и $f(x)$ по заданному модулю $|\tilde{f}(\omega)|$.

Пусть $f(x)$ - интегрируемая в квадрате односторонняя функция, т.е. $f(x)=0$ при $x<0$. Тогда ее преобразование Фурье $f(\omega)=U(\omega)+j V(\omega)-$ предел на действительной оси аналитической в верхней полуплоскости функции $f(z)=U(z)+j V(z)$. В полярных координатах

$$
f(z)=\rho(z) e^{j \varphi(z)} ; \rho=\sqrt{U^{2}+V^{2}} ; \varphi=\operatorname{argtg} \frac{u}{V} ; z=\omega+j \eta
$$

Из уравнений Коши - Ромака

$$
\partial U / \partial \omega=\partial U / \partial \eta=-\partial V / \partial \omega
$$

заменой переменных $U=\rho \cos \varphi, V=\rho \sin \varphi$ с учетом выражения (9) получим

$$
\partial \rho / \partial \omega=\rho \partial \varphi / \partial \eta ; \partial \rho / \partial \eta=-\rho \partial \varphi / \partial \omega .
$$

Производные в уравнениях (10) по условию конечны и непрерывны при $\eta \geq 0$, однако из этого же следует справедливость уравнений (11) при всех $\omega$ и $\eta \geq 0$. При $\rho=0$, в соответствии с (9), переменная $\varphi$ неопределена. Если при некотором $z_{1} f\left(z_{1}\right)=0, \partial \rho\left(z_{1}\right) / \partial \omega$ определена и равна нулю только в том случае, когда все частные производные в уравнениях (10) обращаются в нуль. При отличных от нуля производных в уравнениях (10) производная $\partial \rho / \partial \omega$ имеет разрыв, когда $f(z)=0$. Если $0<\rho<\infty$, то производные $\partial \varphi / \partial \omega, \partial \varphi / \partial \eta$ конечны и непрерывны. Таким образом, уравнения (11) удовлетворяются, если $0<\rho<\infty$ при тех 
значениях $\omega$ и $\eta$, когда справедливы уравнения (10). В этом случае $F(\omega)$ будет аналитической функцией в этой же области.

Чтобы соотношения (7) и (8) имели место, необходимо получить функцию $F(\omega)$ по условиям теорем $1 \div 3$. Если потребовать, чтобы

$$
\int_{-\infty}^{\omega x}|F(\omega)|^{2} d \omega<K
$$

где $\mathrm{K}$ - конечное положительное число, то будет определено поведение $F(\omega)$ при $\omega \rightarrow \infty$. Предположим, что $|F(\omega)|$ убывает достаточно быстро с ростом $\omega$. Но если $\operatorname{Ln}|F(\omega)| \rightarrow 0$ при $|\omega| \rightarrow \infty$, то воспользоваться определением (3) преобразования Фурье $f(x)$ функции $f(\omega)$ можно, только допустив, что $\boldsymbol{f}(\boldsymbol{x})$ содержит дельта-функцию. Из условия $\boldsymbol{F}(\omega) \rightarrow 0$ при $\omega \rightarrow \infty$ следует, что $\tilde{f}(\omega) \rightarrow \mathbf{1}$ при $\omega \rightarrow \infty$, поэтому $f(x)$ имеет особую точку при $x=0$.

На практике всегда используются конечные интервалы для числового представления функции и ее преобразования Фурье. Хотя их значения всюду конечны, характерной особенностью функций, синтезированных с использованием выражений (7) и (8) является то, что верхняя грань sup| $f(x) \mid$ достигается при $x=0$. С учетом предположений относительно поведения $F(\omega)$, когда $\omega \rightarrow \infty$, функция $F(\omega)$ удовлетворяет условиям теоремы, а, следовательно, ее преобразование Фурье обращается в нуль на интервале $(-\infty, 0)$. Этот вывод важен для практической реализации алгоритма синтеза сигнала, поскольку позволяет свести вычисление преобразования Гильберта к операциям преобразования Фурье и фильтрации, определенной соответствующим образом.

Формально можно допустить, что $|\operatorname{Ln} \tilde{f}(\omega)| \rightarrow c$ при $|\omega| \rightarrow \infty$, где $c \neq 0-$ конечное действительное число. Поскольку преобразование Гильберта от постоянной равно нулю, аргумент спектра, вычисленный с помощью выражения (7), соответствует некоторой функции $\tilde{f}_{1}(\omega)$. Очевидно, функции $\tilde{f}(\omega)$ и $\tilde{f}_{1}(\omega)$ отличаются только модулем, и вычисленные значения $\arg \tilde{f}_{1}(\omega)$ соответствуют искомым. Таким образом, использование соотношений (7), (8) при синтезе функций имеет смысл и тогда, когда условие (13) не выполняется, но $\ln f(\omega)$ стремится к конечному пределу при $\omega \rightarrow \infty$.

Пусть $f(x)=0$ при $x<\alpha$ и $\max |f(x)|=|f(\alpha)|$, а преобразование Фурье этой функции $\tilde{f}(\boldsymbol{\omega})$ удовлетворяет условиям теорем (2) и (3). Тогда из выражений (4) и (6) следует, что если $f(\omega) \neq 0$ на действительной оси при всех конечных $\omega$, то $f(\omega+j \eta)$ не обращается в нуль в верхней полуплоскости. В этом случае $L n f(\omega+j \eta)$ - аналитическая в верхней полуплоскости функция, a еe действительная и мнимая части связаны преобразованием Гильберта. Если $f(x)=0$ при $x>\beta$ и $\max |f(x)|=|f(\beta)|$, то логарифм модуля есть аналитическая функция в нижней полуплоскости, и имеют место соотношения (7) и (8) с заменой знака перед интегралом.

Функцию $f(x)$ можно восстановить по заданному модулю преобразования Фурье $[\tilde{f}(\omega)]$, если выполнены указанные условия. Наиболее удобно сформулировать их как частные ограничения, касающиеся функции $f(x)$ и ее преобразования Фурье $\tilde{f}(\omega)$. Если $f(x) \in L_{2}$, то $f(x)$ восстанавливается с использованием соотношения (9) однозначно, когда

$$
\begin{aligned}
& |\tilde{f}(\omega)| \neq 0 \text { при }|\omega|<\infty \\
& \max |f(x)|=|f(0)| ; f(x)=0 \text { при } x<0 .
\end{aligned}
$$

Рассматривая $f(\omega)$ на интервале $-\infty, \infty$, получаем, что при $x=0 f(x)=c \delta(0)$, где $c=\lim _{|\omega| \rightarrow \infty} f(\omega)$.

В более общем случае заданный модуль $|f(\omega)|$ характеризует некоторое множество функций $[f(x)]$, порождаемое сдвигом функции $f(x)$, удовлетворяющей условию (14), по оси $x$. Поэтому можно говорить о восстановлении функции, принадлежащей указанному множеству, для которой выполняются условия (13) и (14). Если задан аргумент комплексного 
спектра и $f(x)$ удовлетворяет условиям (13) и (14), то с помощью соотношения (10) можно синтезировать один из сигналов, принадлежащих множеству $[N f(x)]$, где $N$ - отличное от нуля действительное число.

Большинство встречающихся сигналов и описывающих их функций не удовлетворяет условиям (13) и (14). Однако практически любые сигналы с конечной энергией можно моделировать так, чтобы полученный новый сигнал с точностью до сдвига по оси $x$ описывался функцией, удовлетворяющей условиям (13) и (14), и имелась возможность обратного перехода от модифицированной функции к исходной. Однако, подобные правила модификации применимы и к спектрам опасных сигналов. Аналогично условиям (13) и (14) можно получить требования к структуре модифицированных спектров:

$\tilde{f}(\omega)=0$ при $\omega<\omega_{0}$.

$$
|f(x)| \neq 0, \max |\tilde{f}(\omega)|=\left|\tilde{f}\left(\omega_{0}\right)\right|, \omega_{0} \geq 0 ;
$$

Один из возможных путей модификации состоит в суммировании исходной функции $f(x)$ с некоторой вспомогательной функцией $\psi(x)$, выбранной так, чтобы модифицированная функция $f_{M}(x)=f(x)+\psi(x)$ удовлетворяла условиям (15) и (16). Такое преобразование удобно в случае финитных функций, когда $f(x)=0$ при $x<a, x>b(b>a)$. Здесь возможны следующие правила модификации. Вспомогательную функцию $\psi(x)$ можно выбрать так, что $\psi(x)=0$ при $x<c$, где $c \leq a$, а ее преобразование Фурье $\tilde{\psi}(\omega) \neq 0$ и $|\tilde{\psi}(\omega)|>|\tilde{f}(\omega)|$. При этих условиях модифицированная функция $f_{M}$ восстанавливается с использованием соотношения (9) по модулю $|\tilde{f}(\omega)+\tilde{\psi}(\omega)|$ с $\max \left|f_{M}(x)\right|=\left|f_{M}(0)\right|$.

Выполнить требование $\widetilde{f_{M}}(\omega) \neq 0$ можно и в том случае, когда $f_{M}(x)=0$ при $x>\alpha$, $\alpha \geq b, \max \left|f_{M}(x)\right|=\left|f_{M}(\alpha)\right|$. Теперь $f_{M}(\omega)-$ предел на действительной оси функции $f_{M}(z)$, аналитической в нижней полуплоскости. С помощью соотношения (9) можно найти функцию $f_{M}(y)$, где $y=-x$, которая отличается от исходной началом и направлением отсчета переменной $x$.

Другой возможный путь модификации заключается в весовой обработке финитной функции $f(x)$, если она принимает отличное от нуля значение по крайней мере на одной из границ интервала $[a, b]$, ограничена внутри и равна нулю вне этого интервала. Тогда можно найти такую функцию $P(x)$, чтобы произведение $P(x) f(x)$ удовлетворяло условиям (13) и (14). Очевидно, одна и та же функция $f(x)$ может быть модифицирована различными путями. Двум упомянутым правилам модификации - суммированию с дополнительным известным точно сигналом и весовой обработке финитного сигнала соответствуют эквивалентные операции над их преобразованиями Фурье - суммирование и свертка спектров.

Информацию, достаточную для восстановления модифицированной функции $f_{M}(x)$, содержит не только модуль спектра $\left|\widetilde{f_{M}}(\omega)\right|$, но и функция $\left.A(\omega)=R \mid \widetilde{f_{M}}(\omega)\right]$, где $R-$ любой невырожденный оператор, т.е. справедливо обратное преобразование $\left|\mathrm{f}_{M}(\omega)\right|=R^{-1}[A(\omega)]$. Если в качестве первичных данных о модифицированном сигнале используется аргумент комплексного спектра, то любая функция $B(\omega)=R[\arg f(\omega)]$ сохраняет признаки сигнала, достаточные для его восстановления с точностью до постоянного множителя. Таким образом, модифицированный сигнал может быть интегрирован по модулю, аргументу спектра или по какой-либо из указанных выше функций при известном $R$ путем очередных преобразований с использованием соотношений (9) и (10). Этот путь синтеза опирается на необходимые и достаточные условия, при которых справедливы используемые интегральные преобразования. Наряду с описанным алгоритмом возможны и другие методы восстановления некоторых модифицированных сигналов.

Таким образом, существует подкласс функций, которые могут быть с достаточной точностью восстановлены по известному модулю преобразования Фурье с помощью 
линейных интегральных преобразований. Если задан аргумент комплексного спектра, то функции из этого подкласса восстанавливаются с точностью до последнего множителя. Признаком принадлежности функции $f(x)$ к указанному подклассу является выполнение условий (13) и (14), сформулированных для $f(x)$ и ее преобразования Фурье $f(\omega)$. Эти условия характеризуют поведение $f(x)$ и $f(\omega)$ как функций действительных аргументов $x, \omega$ и поэтому удобны в инженерных приложениях, особенно в радиомониторинге.

Выводы. Пользуясь приведенными признаками можно финитные и односторонние функции с конечной энергией модифицировать, т.е. перевести в изучаемый подкласс с помощью различных, в том числе очень простых, преобразований. Выбор конкретного правила модификации зависит от характера решаемой задачи и особенности преобразования сигналов. Модификация сигналов в ряде случаев расширяет возможности их анализа и обработки. Рассмотренные свойства модифицированных функций согласуются с полученными ранее результатами и дают возможность с общих позиций подойти к изучению большого круга явлений и процессов, в основе описания которых лежат амплитудные или фазовые характеристики.

\section{ЛИТЕРАТУРА}

1. Титчмарш Е. Введение в теорию интегралов Фурье / Титчмарш Е. - М. - Л. ГИТТЛ, $1968-497$ с.

2. Logan B.F. Theory of Analytic Modulation Systems. - The Bell Syst. Tech. J., 57, N 3, p. $491-576$. 